\title{
Erratum
}

Ki Young Lee, Kwang Wook Yoo, Youngsun Choi, Gunpyo Kim, Sangmo Cheon, Jae Woong Yoon* and Seok Ho Song*

\section{Erratum to: Topological guided-mode resonances at non-Hermitian nanophotonic interfaces}

https://doi.org/10.1515/nanoph-2021-0325

After the publication of this article, the authors found out some simple errors and hereby correct them as delineated below.

(1) In lines $21-24$ on page 1856, the previous description on fill factor $F$ is

$\delta$ is defined such that refractive index of the grating bars $n_{\mathrm{c}}=n_{\mathrm{d}}+\delta$ with $n_{\mathrm{d}}=\varepsilon_{\mathrm{d}}^{1 / 2}$ denoting refractive index of the surrounding medium and fill factor $F=\left(n_{\mathrm{c}}-n_{\mathrm{d}}\right) /(2 \delta)$.

This description is incorrect and thereby should be replaced by a correct description as

$\delta$ is defined such that refractive index of the grating bars $n_{\mathrm{c}}=n_{\mathrm{d}}+\delta$ and fill factor $F=\left(n_{\mathrm{a}}-n_{\mathrm{d}}\right) / \delta$, where $n_{\mathrm{d}}=\varepsilon_{\mathrm{d}}{ }^{1 / 2}$ denotes refractive index of the surrounding medium and $n_{\mathrm{a}}=2.864$ is average refractive index of the grating region $0<z<d$.

(2) In line 3-5 on page 1857 , the previous description on topological tuning parameter $\delta$ is

This parameter set corresponds to $\left\{n_{c}=3.385, F=0.442\right\}$ for $\delta=-0.1$ and $\left\{n_{c}=3.585, F=0.365\right\}$ for $\delta=+0.1$.

Here, there is missing parameter $\delta_{\mathrm{EP}}$ in this description and a correct description should be

This parameter set corresponds to $\left\{n_{c}=3.385, F=0.442\right\}$ for $\delta=\delta_{\mathrm{EP}}-0.1$ and $\left\{n_{c}=3.585, F=0.365\right\}$ for $\delta=\delta_{\mathrm{EP}}+0.1$.
*Corresponding authors: Jae Woong Yoon and Seok Ho Song, Department of Physics, Hanyang University, 206, Wangsimni-ro, Seongdong-gu, Seoul, 04763, Korea, E-mail: yoonjw@hanyang.ac.kr (J.W. Yoon) and shsong@hanyang.ac.kr (S. H. Song). https://orcid.org/0000-0002-3362-6873 (J. W. Yoon) Ki Young Lee, Kwang Wook Yoo, Youngsun Choi, Gunpyo Kim and Sangmo Cheon, Department of Physics, Hanyang University, 206, Wangsimni-ro, Seongdong-gu, Seoul, 04763, Korea 